\title{
Epidemiologic, Clinical, Ultrasonographic, and Cytological Features of Thyroid Nodules in Predicting Malignancy Risk: A Retrospective Study of 442 French Afro-Caribbean Patients
}

\author{
Johan Joseph-Auguste, ${ }^{1}$ Lucien Lin, ${ }^{1}$ Magalie Demar, ${ }^{2}$ Olivier Duffas, ${ }^{3}$ Vincent Molinie, ${ }^{4}$ \\ Caroline Sulpicy, ${ }^{5}$ Marie-Josée Dorival, ${ }^{5}$ Olivier Luxembourger, ${ }^{6}$ and Nadia Sabbah $\mathbb{C}^{1,2,7}$ \\ ${ }^{1}$ Department of Endocrinology and Metabolic Diseases, University Hospital Center, Louis Domergues, La Trinité, Martinique \\ ${ }^{2}$ EA3593, Amazon Ecosystems and Tropical Diseases, University of French Guiana, Cayenne, French Guiana \\ ${ }^{3}$ Department of Otorhinolaryngology, University Hospital Center, Pierre Zobda Quitman, Fort-de-France, Martinique \\ ${ }^{4}$ Department of Anatomopathology, University Hospital Center, Pierre Zobda Quitman, Fort-de-France, Martinique \\ ${ }^{5}$ Anatomopathology Center, Schoelcher, Martinique \\ ${ }^{6}$ Department of General Surgery, University Hospital Center, Louis Domergues, La Trinité, Martinique \\ ${ }^{7}$ Department of Endocrinology and Metabolic Diseases, Cayenne Hospital, Andre Rosemon, French Guiana
}

Correspondence should be addressed to Nadia Sabbah; sabbahnadia@yahoo.fr

Received 28 July 2019; Revised 11 November 2019; Accepted 5 February 2020; Published 31 March 2020

Academic Editor: Dario de Biase

Copyright (C) 2020 Johan Joseph-Auguste et al. This is an open access article distributed under the Creative Commons Attribution License, which permits unrestricted use, distribution, and reproduction in any medium, provided the original work is properly cited.

Objective. To evaluate epidemiologic, clinical, cytological, and ultrasonographic features of thyroid nodules in a sample French Afro-Caribbean population to determine if the standard criteria for predicting malignancy risk are applicable to this specific ethnic population. Methods and Design. This retrospectively designed study consisted of 442 patients who had consulted with the Endocrinology Department in Martinique (French overseas department) between 2007 and 2011. Of the 442 patients, 641 ultrasound-guided fine-needle aspirations (US-FNA) were performed by two experienced endocrinologists, and 212 patients underwent surgery. The geographical situation, age, gender of the patient, clinical and ultrasonographic features, TSH level, and US-FNA results were considered and cross-referenced with their pathology results. Results. The overall malignancy rate on final histopathology was $9 \%$ (women only), $80 \%$ of which were papillary cancer, and $20 \%$ were follicular cancer. Occult micropapillary carcinoma represented $35 \%$ of the papillary cancer. There was no significant difference in age, nodule localization, number of nodules, or thyroid function test between benign and malignant nodules. Contrary to the literature, we found only $12 \%$ incidentaloma in our series, while more than half of the nodules were discovered on palpation or as a clinical symptom. Hypoechogenicity in solid pattern nodules and nodules between 2 and $3 \mathrm{~cm}$ in size revealed a high diagnostic value in detecting malignancy. The corresponding rate of malignancy on Bethesda system histopathologic examination was as follows: $0 \%$ in undiagnosed (I), 0\% benign (II) (micropapillary), 5\% (FLUS)/atypia (III), 9\% follicular neoplasm (IV), 33\% suspected malignancy (V), and no malignant cytology (VI). These results show a different Bethesda system predictive value for this French AfroCaribbean population. Conclusion. Studies evaluating ethnic cancer disparities among patients with thyroid cancer are limited and do not specifically focus on the French Afro-Caribbean population. Despite rare thyroid incidentaloma, 35\% of the papillary cancer cases were micropapillary carcinoma, and the incidence and standardized mortality rate in Martinique are lower than in metropolitan France. The malignant risk distribution of thyroid FNA Bethesda classification in this sample population differs from the standard risk, and it is necessary to take that into account in the decision to operate by associating it with echographic malignancy criteria. 


\section{Introduction}

Nodular thyroid disease is a common condition, especially in females and, in particular, among the elderly $[1,2]$. The prevalence of thyroid cancer has increased over the last decade due to earlier detection of thyroid nodules using improved diagnostic imaging techniques. Most thyroid nodules are benign (90-95\%); however, it remains difficult to detect those that may become potentially malignant. Ultrasonography is a noninvasive method that allows the practitioner to distinguish between a malignant nodule and a benign nodule. This method delivers accurate information on the nature, size, topography, echogenicity, microcalcifications, and vascularization of the nodule and therefore improves information for the fine-needle aspiration biopsy (FNAB) [3]. The ultrasound results depend on practitioner's experience. In 2007, there was no official consensus that defined the risk of cancer in relation to the ultrasonographic features of thyroid nodules. However, in 2009, in the American Thyroid Association Guidelines, fourteen sonographic features were mentioned for patients with thyroid nodules [4]. In 2011, a standardized system for analyzing and reporting thyroid ultrasound, the Thyroid Imaging and Reporting Data System (TIRADS), was used to provide initial sonographic assessment of thyroid nodules [5]. In 2017, the new European TIRADS, called EU-TIRADS, was published [6]. FNAB represents the most reliable and noninvasive exam to evaluate the risk of malignancy.

Comparative studies on thyroid cancer or nodule disparities among different ethnic groups are few, and none exist for the members of the Afro-Caribbean population living in their native environment. Several studies evaluated ethnic disparities among patients with thyroid cancer, and a few of them described clinical, epidemiological, ultrasonographic, and cytological malignancy risk in the AfroAmerican population [7, 8].

Environmental exposure, as well as diet and lifestyle, is known to initiate epigenetic changes. Epigenetic research in the field of thyroid cancer has contributed to improving our understanding of biologic determinants in cancer disparities [9]. Finalyson et al. described, among his study pool in England, a higher risk of thyroid cancer in black Africans and black Caribbeans compared to the white population (in particular for follicular thyroid cancer) [10].

Some studies have described a lower rate of thyroid cancer in Afro-Americans, and the data come from the US National Cancer Institute's Surveillance, Epidemiology, and End Results database from 1988 to 1992 and from 1992 to $2010[11,12]$. Magreni et al. observed no significant difference in the incidence of thyroid cancer between whites and blacks and social determinants, and access to healthcare did not seem to be the major factor [13]. Most trials took into account racial and socioeconomic disparities [14], but few focused on epidemiologic ultrasonographic and histological differences. Moo-Young et al. showed that Afro-American patients $<45$ years had larger thyroid tumors but were less likely to have lymph node invasion [15]. Healthcare in Martinique is accessible to all and is therefore not a differentiation factor as it is in America. Martinique is a French
Caribbean Island with 394,000 inhabitants and different ethnicities: $75 \%$ of the population is Afro-Caribbean and $25 \%$ is Caucasian. The aim of this study was to gain greater insight into potential disparities concerning the malignancy risk factors among the local Afro-Caribbean population and to describe the related epidemiologic, clinical, ultrasound, and cytological factors. The secondary objective was to analyze the cytological and histopathological correlations to determine if the Bethesda system could be applicable to this ethnic group. The results of FNAB and the ultrasonographic features were correlated with the final histopathology diagnosis after surgery. The frequency of malignancy was determined and compared with the national database.

\section{Subjects and Methods}

This study is a retrospective trial, and the study pool consisted of 530 patients referred to the Department of Endocrinology, University Hospital Center in La Trinité, Martinique. This included all patients who presented with thyroid nodules and underwent FNAB in our center between 2007 and 2009. Only two centers performing FNAB exist in Martinique. The FNAB were performed for all nodules. The criteria to refer patient in our center for ultrasound-guided FNAB were a nodule larger than $8 \mathrm{~mm}$ or suspicious malignant sonographic features such as microcalcification, irregular margin, markedly hypoechogenicity, and increased central vascularity.

Eighty-eight patients were excluded due to cytology or histopathology reports that were unavailable. Thyroid US and US-FNA were performed with $5-12 \mathrm{MHz}$ linear array transducers (HD11, Philips-Advanced Technology Laboratories) by two specialized radiologists with over ten years of thyroid imaging experience. Ultrasonographic features were determined: nodule size, echo structure (solid, cystic, and mixed), echogenicity, margin (well-defined regular halo or poorly defined irregular), and presence or absence of microcalcifications. The vascularization was none, type I; presence of perinodular and/or slight intranodular flow, type II; and presence of perinodular and/or slight intranodular flow, type III.

Patients signed a consent form prior to the procedure. FNAB was performed after local anesthesia with xylocaine $1 \%$ gel and with antiseptic technique. The thyroid lesions were sampled from a single skin puncture with a $25 \mathrm{G}$ needle and, if necessary, a syringe $(10 \mathrm{~mL})$. Two to three samplings were performed per nodule, and the sample was air-dried. Slides prepared from each nodule were stained and fixed with alcohol and then submitted for Papanicolaou staining. When the nodule had cystic and solid components, both components were sampled. Histopathologic diagnosis was performed by four different cytopathologists during the study period. All the FNAB results were classified using the Bethesda System for Reporting Thyroid Cytopathology 2009 $[16,17]$. For the FNAB performed prior to the Bethesda system, a cytopathologist reviewed and classified these samples using the Bethesda system (Bethesda classification analyzes the malignancy risk for each category: I, nondiagnostic or unsatisfactory (ND/UNS); II, benign; III, 
atypia of undetermined significance (AUS)/follicular lesion of undetermined significance (FLUS); IV, follicular neoplasm/suspicious for follicular neoplasm; IV, suspicious for malignancy; V, malignant). Histological examination was performed for nodules with clinical suspicion, those suspected by cytology, and patients with two consecutive AUS/ follicular neoplasm diagnoses, as well as for nodules with strong suspicion of ultrasound and those measuring more than $3 \mathrm{~cm}$ (after agreement of the multidisciplinary consultation meeting). If a focus of microcarcinoma was found outside of the nodule of interest, it was considered clinically insignificant and was excluded for determining the predictive value of the FNAB. The following data were recorded for all the patients in the study: gender, age, residence, BMI, thyroid dysfunction, number and size of thyroid nodule, ultrasound features, thyroid stimulating hormone ultrasensitive (TSHus), and FNAB results (Bethesda system). Comparisons were performed to determine the predictive factors of malignancy. The quantitative data were obtained by calculating the mean and standard deviation. We used a student test for correlation. The qualitative data were obtained by calculating the percentage and absolute value, and comparisons were performed with a Fisher exact test for categorical variables. $p<0.05$ was considered statistically significant.

Clinical and ultrasonographic features were evaluated using univariate and multivariate analyses with logistic regression.

2.1. Ethical Approval. The local ethics committee agreed, and oral consent was obtained by practitioners according to European regulations. The declaration of use of the data number 2214515 of the National Commission of Informatics and Freedoms was obtained.

\section{Results}

Between 2007 and 2009, a total of 530 patients underwent thyroid US with FNAB (exclusion criteria: nodule $<8 \mathrm{~mm}$ ); 88 patients were excluded from the analysis: 5 patients refused to be operated on, and for 83 patients, histological and operating reports were unavailable. We studied 442 patients for a total of 641 thyroid nodules. Most patients were referred by their primary care physician (66\%). Of the 442 patients, 329 had an informed mode of discovery. Unlike most studies, we primarily had a clinical mode of discovery, either with palpation or during presentation of symptoms such as genes or dyspnea. In fact, this represents more than $58 \%$ of the patients in our study ( $20 \%$ had a thyroid history and had a follow-up). The incidentalomas represent only $12 \%$ (with those followed for parathyroid disease) of the nodules discovered, and 7\% of those patients had TSH abnormalities. Among the 12 cancers, 8 were discovered on clinical signs or on palpation, 2 were incidentalomas, and 2 were nodules followed previously. Only $10 \%$ had a chance discovery after another examination, $2 \%$ were discovered during a primary hyperparathyroidism follow-up (so $12 \%$ of total incidentaloma), and 6\% were discovered during a cervical ultrasound performed for biological dysthyroidism. Half of the patients (48\%) were operated on to control 264 nodules and 212 patients; $22 \%$ benefited from a lobectomy and $78 \%$ from a complete thyroidectomy. Lobectomy was proposed to patients with a single nodule that was benign in cytology but had increased significantly in size (more than $20 \%$ in the year) or measuring more than $3 \mathrm{~cm}$ for Bethesda III (after two FNAB) or IV isolated nodules. Thyroidectomy was performed for voluminous multinodular goiter, suspicious nodule with contralateral lesion, or during lobectomy if the lesion was suspicious in extemporaneous.

The operated patients were similar to the sample patients for age, gender, TSHus rate, and personal and familial thyroid history. The body mass index (BMI) medium for operated patients with benign disease was 28 , and for patients with thyroid cancer, it was 31 . The patients who underwent surgery were women, who were on average 50 years old with no thyroid history. None of the patients had any familial thyroid cancer history. Most patients were female (87\%), and malignant nodules were only associated with the female gender. Among the operated patients, we found a malignancy rate of $9 \%(n=19)$. Microcancers were incidentally detected after thyroidectomy for another nodule in 3\%. These occult micropapillary carcinomas represented $35 \%(n=7)$ of the cancers. Among the cancers, $80 \%$ were papillary carcinomas, and $20 \%$ were follicular carcinomas (one microfollicular).

Patients with thyroid cancer were not significantly different from those with benign nodules $(p>0.05)$. The thyroid cancer rate increased with age until an average age of 50 years in each histological type (Table 1). Overall, 25\% $(n=5)$ had a benign thyroid history, $10 \%(n=2)$ had a malignant transformation (these 2 cases were those of two patients who were considered to have repeatedly benign nodules by FNAB, who were followed during 5 years for one and 4 years for the second, and in whom FNAB finally found lesions of the follicular neoplasm type justifying surgery and the ultimate diagnosis of thyroid cancer (papillary carcinoma)), $15 \%(n=3)$ had a benign nodule history, and $50 \%$ had no thyroid disease history.

3.1. Ultrasound Description. The majority of operated goiters were $64 \%(n=163)$, including nodules superior at $3 \mathrm{~cm}, 71 \%$ $(n=150)$ were plurinodular, and $29 \%(n=62)$ were isolated nodules. Of these, $65 \%(100 / 153$ indicated values) had a solid echo pattern, $31 \%$ (48/153 indicated values) had a mixed echo pattern, and $11 \%(22 / 194$ indicated values $)$ had hypervascularization type III. Of the patients, $63 \%(141 / 223$ indicated values) were hypoechogenic, 20\% (53/262 indicated values) had irregular borders, and 20\% (54/264 indicated values) had microcalcifications (Table 2). At the time of the study, the TIRADS classification was not still in use, and in the data alone, the echogenicity, irregularity of the margins, vascularization, and the presence of microcalcification were described. There was little information in the records about height and width, as well as the importance of hypoechogenicity. Thus, we could not reclassify the nodules in TIRADS but only describe the echogenicity, the 
TABLE 1: Characteristics of the patients who underwent surgery.

\begin{tabular}{lcc}
\hline Variables & Patients without surgery $\left(n^{*}=442\right)$ & Patient with surgery $\left(n^{*}=212\right)$ \\
\hline Age (years) & $51 \pm 14$ & $50 \pm 13$ \\
Sex & & $185(87 \%)$ \\
Women & $398(90 \%)$ & $27(13 \%)$ \\
Men & $44(10 \%)$ & 0.6 \\
Family history & $414(94 \%)$ & $198(93 \%)$ \\
None & $28(6 \%)$ & $14(7 \%)$ \\
Nodule & $0(0 \%)$ & $0(0 \%)$ \\
Thyroid cancer & & $168(80 \%)$ \\
Personal thyroid history & $354(80 \%)$ & $15(7 \%)$ \\
None & $44(9 \%)$ & $19(9 \%)$ \\
Cytology & $34(8 \%)$ & $7(3 \%)$ \\
Previous surgery & $14(3 \%)$ & $1.2 \pm 2.3$ \\
Thyroid disease & $1.3 \pm 2$ & 0.8 \\
TSH & $139(31 \%)$ & $65(31 \%)$ \\
Geographical distribution & $95(21 \%)$ & $38(18 \%)$ \\
Center & $189(43 \%)$ & $94(45 \%)$ \\
South & $12(3 \%)$ & $6(3 \%)$ \\
North-Atlantique & & 0.7 \\
North-Caribbean & & 0.8 \\
\hline
\end{tabular}

* Number of nodules.

TABLE 2: Ultrasound characteristics: echogenicity, irregular margin, and microcalcification in 414 thyroid cancer and benign lesions.

\begin{tabular}{|c|c|c|c|c|c|}
\hline & & Histology benign $(n=261)$ & Histology malignant $(n=12)$ & $n$ & $p$ \\
\hline \multirow{3}{*}{ Hypoechogenicity, $n$} & Iso & $73(35 \%)$ & $2(17 \%)$ & 75 & 0.22 \\
\hline & Hyper & $6(2.8 \%)$ & $1(8.3 \%)$ & 7 & - \\
\hline & Hypo & $132(63 \%)$ & $9(75 \%)$ & 141 & - \\
\hline \multirow{2}{*}{ Irregular margin, $n$} & No & $201(80 \%)$ & $6(55 \%)$ & 207 & \\
\hline & Yes & $48(20 \%)$ & $5(45 \%)$ & 53 & 0.05 \\
\hline \multirow{2}{*}{ Microcalcifications, $n$} & Yes & $51(20 \%)$ & $3(25 \%)$ & 54 & 0.71 \\
\hline & No & $203(80 \%)$ & $9(75 \%)$ & 212 & - \\
\hline
\end{tabular}

limits, the presence of calcifications, and the type of vascularization. Tumors with sizes $<3 \mathrm{~cm}$ and $>2 \mathrm{~cm}$ were significantly more prevalent for a malignant nodule $(p<0.05)$. Plurinodular goiters were not associated with a cancer risk. The ultrasound criteria of malignancy that stood out from the univariate logistic regression were the irregular borders $(p<0.05)$ (Table 2$)$.

\section{FNAB}

Our cytology results found $45 \%$ of tumors were benign cytology (Bethesda II), 16\% were atypia of undetermined significance (Bethesda III), 27\% were follicular neoplasms (Bethesda IV), and 5\% were suspected of malignancy (Bethesda $\mathrm{V}$ ) with respective risk of malignancy of $0 \%, 5 \%$, $9 \%$, and $33 \%$, respectively (Table 3 ). We did not find malignant cytology (Bethesda VI). Among the operated patients who underwent FNAB, $14 \%$ of the cytologies were not contributory, and mostly solid nodules were found. Of these, none were malignant, and $81 \%$ of these cytologies corresponded to nodules larger than $3 \mathrm{~cm}$. Cytological results did not agree with histological results in $11 \%$ of cases. Of these cytological discordances, $60 \%$ were nodules larger than $3 \mathrm{~cm}$. The reliability of cytology decreased with nodule size (Table 4).
In thyroid cancer nodules (excluded occult micropapillary) with suspicious malignancy cytology (Bethesda $\mathrm{V})$, hypoechogenicity was found in $75 \%$ of tumors, irregular margin was found in $25 \%$, microcalcifications were found in $16 \%$, and vascularization III was found in 33\%. The concordance between Bethesda classification and ultrasound criteria in thyroid cancer is described in Table 5. The cytology in our study had a sensitivity of $83 \%$ and a specificity of $72 \%$ (noncontributive cytology was included, and occult micropapillary carcinomas were excluded from the results).

\section{Discussion}

The incidence of thyroid cancer has increased since the 1990 s. It is now the 5th most common cancer in women, whereas 20 years ago, it was 14 th $[18,19]$. The incidence of thyroid cancer in Martinique is 2.2 for men and 8.6 for women per 100,000 inhabitants; in metropolitan France, the figures are 4.89 for men and 14.8 for women, per 100,000 inhabitants. The standardized mortality rate is also lower, at 0.2 and 0.25 for Martinique and metropolitan France, respectively (data from the general cancer registry of Martinique). Our results confirm previous studies showing a different incidence and survival among people of different racial and ethnic backgrounds, such as study by Morris et al., 
TABLE 3: Malignancy risk and Bethesda classification.

\begin{tabular}{lcccc}
\hline $\begin{array}{l}\text { Cytological diagnosis Bethesda } \\
\text { classification }\end{array}$ & $\begin{array}{c}\text { All FNAB, } N \\
(\%)\end{array}$ & $\begin{array}{c}\text { All FNAB with surgical follow-up, } \\
N(\%)^{*}\end{array}$ & $\begin{array}{c}\text { Malignancy risk, } \begin{array}{c}N \\
(\%)^{* * *}\end{array} \\
\begin{array}{c}\text { Benign histology, } \\
(\%)\end{array}\end{array}$ \\
\hline Benign II & $347(55 \%)$ & $111(45 \%)$ & $0(0 \%)$ & $111(100 \%)$ \\
AUS/FLUS III & $60(9.5 \%)$ & $41(16 \%)$ & $2(5 \%)$ & $39(94 \%)$ \\
Follicular neoplasm IV & $79(12.5 \%)$ & $57(27 \%)$ & $5(9 \%)$ & $52(91 \%)$ \\
Suspicious of malignancy V & $15(2.5 \%)$ & $15(5 \%)$ & $5(33 \%)$ & $10(66 \%)$ \\
Nondiagnostic I & $115(18.5 \%)$ & $36(14 \%)$ & $0(0 \%)$ & $36(100 \%)$ \\
Total & 616 & 260 & 12 & 248 \\
\hline
\end{tabular}

${ }^{*}$ Number and percentage of cases operated in each diagnostic category; ${ }^{* *}$ number and percentage of cases calculated from the total number of operated cases in each category. AUS/FLUS: atypia of undetermined significance or follicular lesion of undetermined significance.

TABLE 4: Concordance between noncontributory FNAB and nodule size.

\begin{tabular}{lccc}
\hline FNAB & Concurring & No concurring & Non contributory \\
\hline Nodule size $(\mathrm{cm})$ & & & \\
$<2$ & $17(6 \%)$ & $6(2 \%)$ & $2(1 \%)$ \\
$2-3$ & $60(22 \%)$ & $6(2 \%)$ & $5(2 \%)$ \\
$>3$ & $121(44 \%)$ & $18(7 \%)$ & $29(11 \%) p<0.03$ \\
\hline
\end{tabular}

TABLE 5: Concordance between Bethesda and echogenicity in the thyroid cancer group.

\begin{tabular}{|c|c|c|c|c|c|}
\hline Bethesda classification & Hypoechogenicity* $^{*}$ & Irregular margin ${ }^{* *}$ & Calcifications s** $^{* *}$ & Vascularization type III**** & Total cancer \\
\hline Benign II & 0 & 0 & 0 & 0 & 0 \\
\hline ASI/LFI III & $2(100 \%)$ & 0 & $1(50 \%)$ & $1(50 \%)$ & 2 \\
\hline Follicular neoplasm IV & $3(60 \%)$ & $1(20 \%)$ & 0 & 0 & 5 \\
\hline Suspicious of malignancy $\mathrm{V}$ & $4(80 \%)$ & $2(40 \%)$ & $1(20 \%)$ & $3(60 \%)$ & 5 \\
\hline Nondiagnosed I & 0 & 0 & 0 & 0 & 0 \\
\hline Total & $9(75 \%)$ & $3(25 \%)$ & $2(16 \%)$ & $4(33 \%)$ & $12(100 \%)$ \\
\hline
\end{tabular}

${ }^{*}$ Number of nodules in each category of echogenicity; ${ }^{* *}$ number of nodules in each category with irregular margins; ${ }^{* * *}$ number of nodules in each category with calcifications; ${ }^{* * * *}$ number of nodules in each category with vascularization type III. AUS/FLUS: atypia of undetermined significance or follicular lesions of undetermined significance.

who described a lower incidence of thyroid cancer for the Afro-American population [20]. Tang et al. in 2018 showed no differences for disease-specific survival but different risk factors between black and white patients. In this study black Americans had less lymph node metastasis of classical variant papillary thyroid carcinoma and follicular variant papillary thyroid carcinoma but not follicular thyroid carcinoma and had a higher risk of distant metastasis for a follicular variant papillary thyroid carcinoma [21]. A recent study described a higher thyroid cancer incidence in the black population, which was due principally to an increased risk of follicular cancer. In contrast, among the Chinese population, the main risk was papillary cancer [10]. Currently, the etiologies of the global thyroid cancer increase remain unknown, but environmental and hormonal influences are possible factors, even if the increase in detection is partly due to the rise of incidentaloma screening, thanks to advances in ultrasound and CT imaging. In our study, most patients were females (87\%), and malignant nodules were only associated with females. In the literature, some studies point to a potential risk of thyroid cancer in obese patients $[22,23]$. In our study, the average BMI in operated men was 25 , while women's BMI was 28 , and for those with benign histology and those with cancer, it was 31. Unfortunately, data about weight and height were incomplete, so only 130 of 220 surgical patient charts were informed. In 2019, Kwon et al. described a higher risk of thyroid cancer in obese men and excessive adiposity per se as an independent risk factor, while women have a greater risk only when obesity is associated with metabolic abnormalities [24]. The PODIUM study showed a very high prevalence of women's obesity: $27 \%$ versus $16 \%$ for men in Martinique, which could be one hypothesis for this high female prevalence [25]. In our region, unlike other French departments, the discovery of nodules is mainly clinical, and they are detected in the course of treating a different complaint, with cervical palpation or complaint (cough, pharyngeal discomfort, voice modification, or lymphadenopathy) near 50\%. Brito et al. showed that, between 1935 and 2012, thyroid cancer in Olmsted County, Minnesota, had an increased incidence of thyroid cancer to 7.1 per 100,000 persons per year between 1990 and 1999 and 13.7 per 100,000 persons per year during 2000-2012, principally due to the increase in occult cancers accidentally discovered ( $20 \%$ of cases), but disease mortality was unchanged [26]. The radiological prevalence of incidentaloma in Martinique is low (10\% incidentaloma in our study versus 55\% in metropolitan France) [27]. This explains the late discovery of nodules and the fact that more than half of them measured over $3 \mathrm{~cm}$ (61\%). This delay could be related to the difficult access to radiological examinations in Martinique, due to the limited number of radiologists ( 9 per 100000 inhabitants in Martinique and 11 per 100000 
inhabitants for France hexagonal) and a smaller number of endocrinologists (2 per 100000 inhabitants compared to France hexagonal 2.6 per 100000) http://www.data.drees. sante.gouv.fr. This is contrary to what is described by Uldelsman and Zhang, who linked the increase in the incidence of thyroid cancer to the increase in endocrinologists and surgeons in the United States who perform ultrasonography and subsequently FNAB, more often [28]. As described by Reitzel et al. [29], it is important to limit the indications of thyroid cancer, especially in populations where access to care is easy and where the overdiagnosis of thyroid incidentaloma is responsible for the increase in thyroid cancer. In 2006, an American team published a study on the risk of malignancy in different populations according to the social criteria. It found a bias in the analysis of the prevalence of thyroid cancer in populations based on ease of access to the healthcare system, such as the socially disadvantaged $[30,31]$. In addition, the patient's noncompliance with different screening campaigns could also be involved. In our study, access to care is the same for the majority of the population because all patients have access to social insurance. However, there may be a bias due to the access to specialists, which is more complicated for residents of municipalities located far from major cities and who have little public transport. Despite this delay in access to healthcare, we observed that, in our population, papillary cancer is more frequent. We suppose that the higher iodine levels on our island could contribute to this result (no data exist for French Caribbean islands on median urinary iodine concentrations). Insufficient iodine in the diet is associated with an increase in the risk of follicular thyroid cancer; in contrast, a diet high in iodine, such as in the Caribbean islands, has been associated with an increased risk in papillary thyroid cancer [32]. We observed $35 \%$ of incidental micropapillary cancers (as defined by the WHO, any thyroid papillary carcinoma of less than $1 \mathrm{~cm}$ in diameter) [33]. Currently, there is no clear definition for incidental thyroid carcinoma, and thus, we observed a very different percentage than the literature [34]. Most patients have a very good prognosis, and the outcome for these patients is excellent. Many micropapillary cancers were discovered during the autopsy of patients who died from an etiology other than thyroid cancer $[35,36]$.

In our study, we obtained a thyroid FNAB in the group of patients who underwent an operation and classified them using the Bethesda system nondiagnostic (I) 36 (14\%), benign (II) 111 (45\%), atypia of undetermined significance/ follicular lesion of undetermined significance (III) 41 (16\%), follicular neoplasm/suspicious for a follicular neoplasm (IV) 57 (27\%), suspicious for malignancy (V) 15 (5\%), and no malignancy (Table 3). We observed a different predictive malignant risk for thyroid FNAB in the Afro-Caribbean population using the Bethesda system (Table 3). A metaanalysis by Sheffield et al. demonstrated a wide variety of observed nodule malignancy risks, particularly for Bethesda III and Bethesda IV [37]. Archuletta et al. found no significant differences in the risk evaluation for individual Bethesda categories between Afro-Americans and nonAfro-Americans but observed a higher number of cases in
Bethesda III. They described more follicular carcinoma and less papillary carcinoma than in the white population and larger tumors at initial diagnosis [7], but Bresler et al. found that the nodule's malignancy risk for Bethesda III and Bethesda IV was nearly twice the expected risk based on original Bethesda system reports [8]. Royer et al. described a source of artifacts from ultrasound gel medium, which could lead to misdiagnosis, but in our study, we used probe cover to limit this risk [38]. We have noticed two nodules with malignant transformation. Obviously, this could have been a repeated false diagnosis of benign lesion, while it was in fact already a malignant. However, such malignant transformation of a benign nodule has already been described in the literature particularly because follicular adenomas and Hürthle cell adenomas have similarities with both benign and malignant tumors, suggesting that some of these lesions are premalignant. They usually have a slow and favorable evolution [39]. A new entity described in 2016, the noninvasive follicular thyroid neoplasm with papillary-like nuclear features, which is a subset of follicular variant of papillary thyroid carcinoma with an indolent evolution [40] could also have been evoked. Unfortunately, in our study, we could not do a screening to search for noninvasive follicular thyroid neoplasm with papillary-like nuclear features, because the period of time between the publication and the pathology was too long, which might have changed the malignancy rate in our study [41]. Concerning the absence of Bethesda VI cytology specimens, it could be due to the fact that the diagnoses were performed by general pathologists and not by specialized cytopoathologist.

Concerning the weak points of this study, our data are not fully representative of the total population, and the results could be biased due to the low proportion of patients from the North Caribbean. In addition, information on education, drinking and smoking habits, occupation, and occupational and recreational exposure to chemicals is incomplete, and information about the nodule shape (taller than wide) for nodule malignancy risk evaluation was not available, so TIRADS classification was not applicable. The small number of cancers limits the statistical analysis; in fact, we mainly note the hypoechogenicity as an echographic criterion almost always present (75\% of thyroid cancer), whereas the irregular limits or microcalcifications are present, respectively, only in 25 and $16 \%$ (Table 5). Another limit to our study is the time elapsed from 2009 to the present; however, the population in our island has remained stable and the environmental factors and the situation of the number of radiologists and specialists have not changed.

\section{Conclusion}

Clinical, cytological, and ultrasonography characteristics of thyroid nodules to evaluate the risk of thyroid cancer malignancy in different racial groups cannot be clearly identified. In this French Afro-Caribbean population, some elements have been highlighted: the incidence among women is greater than in other departments, micropapillary and papillary carcinoma predominate, and the risk assessment of malignancy by the Bethesda system is less reliable 
for groups IV and V. The prevalence of thyroid cancer in Martinique is lower, with a mortality rate identical to that of other French departments. Thus, it is intriguing to note that symptoms and clinical examinations remain the preferred mode of screening and avoid having to question the management of incidentalomas.

\section{Abbreviations}

TIRADS: Thyroid Imaging and Reporting Data System FNAB: $\quad$ Fine-needle aspiration biopsy

ND/UNS: Nondiagnostic or unsatisfactory

AUS: $\quad$ Atypia of undetermined significance

FLUS: Follicular lesion of undetermined significance

TSHus: Thyroid stimulating hormone ultrasensitive

BMI: $\quad$ Body mass index.

\section{Data Availability}

The data that support the findings of this study are available from the corresponding author upon reasonable request.

\section{Conflicts of Interest}

The authors declare that they have no conflicts of interest.

\section{Acknowledgments}

The authors express their gratitude to Pr. P. Chanson and Pr. Leenhart for their outstanding support and Dr. C. JoachimContaret for thyroid cancer data in Martinique.

\section{References}

[1] G. H. Tan and H. Gharib, "Thyroid incidentalomas: management approaches to nonpalpable nodules discovered incidentally on thyroid imaging," Annals of Internal Medicine, vol. 126, no. 3, pp. 226-231, 1997.

[2] L. Hegedüs, "The thyroid nodule," New England Journal of Medicine, vol. 351, no. 17, pp. 1764-1771, 2004.

[3] G. Anil, A. Hegde, and F. H. V. Chong, "Thyroid nodules: risk stratification for malignancy with ultrasound and guided biopsy," Cancer Imaging, vol. 11, pp. 209-223, 2011.

[4] D. S. Cooper, G. M. Doherty, B. R. Haugen et al., "Revised American thyroid association management guidelines for patients with thyroid nodules and differentiated thyroid cancer," Thyroid, vol. 19, no. 11, pp. 1167-1214, 2009.

[5] G. Russ, C. Bigorgne, B. Royer, A. Rouxel, and M. BienvenuPerrard, "Le système TIRADS en échographie thyroïdienne," Journal de Radiologie, vol. 92, no. 7-8, pp. 701-713, 2011.

[6] G. Russ, S. J. Bonnema, M. F. Erdogan, C. Durante, R. Ngu, and L. Leenhardt, "European thyroid association guidelines for ultrasound malignancy risk stratification of thyroid nodules in adults: the EU-TIRADS," European Thyroid Journal, vol. 6, no. 5, pp. 225-237, 2017.

[7] P. A. Archuletta, R. Gidwani, M. Husain et al., "The Bethesda System thyroid diagnostic categories in the African-American population in conjunction with surgical pathology follow-up," CytoJournal, vol. 9, p. 7, 2012.

[8] A. Bresler, V. Mehta, B. A. Schiff et al., "Comparison of Bethesda cytopathology classification to surgical pathology across racial-ethnic groups," Head \& Neck, vol. 41, no. 7, pp. 2340-2345, 2019.

[9] B. E. Henderson, N. H. Lee, V. Seewaldt, and H. Shen, "The influence of race and ethnicity on the biology of cancer," Nature Reviews Cancer, vol. 12, no. 9, pp. 648-653, 2012.

[10] A. Finlayson, I. Barnes, S. Sayeed, B. McIver, V. Beral, and R. Ali, "Incidence of thyroid cancer in England by ethnic group, 2001-2007," British Journal of Cancer, vol. 110, no. 5, pp. 1322-1327, 2014.

[11] Racial-ethnic-monograph.pdf, https://seer.cancer.gov/archive/ publications/ethnicity/racial-ethnic-monograph.pdf.

[12] A. Magreni, D. V. Bann, J. R. Schubart, and D. Goldenberg, "The effects of race and ethnicity on thyroid cancer incidence," JAMA Otolaryngology-Head \& Neck Surgery, vol. 141, no. 4, pp. 319-323, 2015.

[13] Racial/Ethnic Patterns of Cancer in the U.S., 1988-1992-SEER Publications, SEER, https://seer.cancer. gov/archive/publications/ethnicity/index.html.

[14] A. Harari, N. Li, and M. W. Yeh, "Racial and socioeconomic disparities in presentation and outcomes of well-differentiated thyroid cancer," The Journal of Clinical Endocrinology \& Metabolism, vol. 99, no. 1, pp. 133-141, 2014.

[15] T. A. Moo-Young, J. Panergo, C. E. Wang et al., "Variations in clinicopathologic characteristics of thyroid cancer among racial ethnic groups: analysis of a large public city hospital and the SEER database," The American Journal of Surgery, vol. 206, no. 5, pp. 632-640, 2013.

[16] E. S. Cibas and S. Z. Ali, "The bethesda system for reporting thyroid cytopathology," Thyroid, vol. 19, no. 11, pp. 1159-1165, 2009.

[17] Z. W. Baloch, V. A. LiVolsi, S. L. Asa et al., "Diagnostic terminology and morphologic criteria for cytologic diagnosis of thyroid lesions: a synopsis of the national cancer institute thyroid fine-needle aspiration state of the science conference," Diagnostic Cytopathology, vol. 36, no. 6, pp. 425-437, 2008.

[18] R. Vigneri, P. Malandrino, and P. Vigneri, "The changing epidemiology of thyroid cancer," Current Opinion in Oncology, vol. 27, no. 1, pp. 1-7, 2015.

[19] C. La Vecchia, M. Malvezzi, C. Bosetti et al., "Thyroid cancer mortality and incidence: a global overview," International Journal of Cancer, vol. 136, no. 9, pp. 2187-2195, 2015.

[20] L. G. T. Morris, A. G. Sikora, D. Myssiorek, and M. D. DeLacure, "The basis of racial differences in the incidence of thyroid cancer," Annals of Surgical Oncology, vol. 15, no. 4, pp. 1169-1176, 2008.

[21] J. Tang, D. Kong, Q. Cui et al., "Racial disparities of differentiated thyroid carcinoma: clinical behavior, treatments, and long-term outcomes," World Journal of Surgical Oncology, vol. 16, no. 1, 2018.

[22] S.-K. Myung, C. W. Lee, J. Lee, J. Kim, and H. S. Kim, "Risk factors for thyroid cancer: a hospital-based case-control study in Korean adults," Cancer Research and Treatment, vol. 49, no. 1, pp. 70-78, 2017.

[23] D. Schmid, C. Ricci, G. Behrens, and M. F. Leitzmann, "Adiposity and risk of thyroid cancer: a systematic review and meta-analysis," Obesity Reviews, vol. 16, no. 12, pp. 1042-1054, 2015.

[24] H. Kwon, Y. Chang, A. Cho et al., "Metabolic obesity phenotypes and thyroid cancer risk: a cohort study," Thyroid, vol. 29, no. 3, pp. 349-358, 2019.

[25] J.-L. Daigre, A. Atallah, J.-L. Boissin et al., "The prevalence of overweight and obesity, and distribution of waist circumference, in adults and children in the French overseas 
territories: the PODIUM survey," Diabetes \& Metabolism, vol. 38, no. 5, pp. 404-411, 2012.

[26] J. P. Brito, A. Al Nofal, V. M. Montori, I. D. Hay, and J. C. Morris, "The impact of subclinical disease and mechanism of detection on the rise in thyroid cancer incidence: a population-based study in olmsted county, Minnesota during 1935 through 2012," Thyroid, vol. 25, no. 9, pp. 999-1007, 2015.

[27] J.-L. Wémeau, J.-L. Sadoul, Herbomez et al., "Recommandations de la Société française d'endocrinologie pour la prise en charge des nodules thyroïdiens," Medicine, vol. 40, no. 9, 2019.

[28] R. Udelsman and Y. Zhang, "The epidemic of thyroid cancer in the United States: the role of endocrinologists and ultrasounds," Thyroid, vol. 24, no. 3, pp. 472-479, 2014.

[29] L. R. Reitzel, N. Nguyen, N. Li, L. Xu, S. D. Regan, and E. M. Sturgis, "Trends in thyroid cancer incidence in Texas from 1995 to 2008 by socioeconomic status and race/ethnicity," Thyroid, vol. 24, no. 3, pp. 556-567, 2014.

[30] L. Davies, L. G. T. Morris, M. Haymart et al., "American association of clinical endocrinologists and American college of endocrinology disease state clinical review: the increasing incidence of thyroid cancer," Endocrine Practice, vol. 21, no. 6, pp. 686-696, 2015.

[31] S. Altekruse, A. Das, H. Cho, V. Petkov, and M. Yu, "Do US thyroid cancer incidence rates increase with socioeconomic status among people with health insurance? an observational study using SEER population-based data," BMJ Open, vol. 5, no. 12, Article ID e009843, 2015.

[32] F. Delange, "Risks and benefits of iodine supplementation," The Lancet, vol. 351, no. 9107, pp. 923-924, 1998.

[33] C. Hedinger, E. Dillwyn Williams, and L. H. Sobin, "The WHO histological classification of thyroid tumors: a commentary on the second edition," Cancer, vol. 63, no. 5, pp. 908-911, 1989.

[34] R. Reinke, J. S. Mathiesen, S. R. Larsen et al., "Incidental and non-incidental papillary thyroid microcarcinoma in Denmark 1996-2015: a national study on incidence, outcome and thoughts on active surveillance," Cancer Epidemiology, vol. 60, pp. 46-50, 2019.

[35] I. D. Hay, M. E. Hutchinson, T. Gonzalez-Losada et al., "Papillary thyroid microcarcinoma: a study of 900 cases observed in a 60-year period," Surgery, vol. 144, no. 6, pp. 980-988, 2008.

[36] S. Noguchi, H. Yamashita, S. Uchino, and S. Watanabe, "Papillary microcarcinoma," World Journal of Surgery, vol. 32, no. 5, pp. 747-753, 2008.

[37] B. S. Sheffield, H. Masoudi, B. Walker, and S. M. Wiseman, "Preoperative diagnosis of thyroid nodules using the bethesda system for reporting thyroid cytopathology: a comprehensive review and meta-analysis," Expert Review of Endocrinology \& Metabolism, vol. 9, no. 2, pp. 97-110, 2014.

[38] M. C. Royer, D. D. Davidson, R. K. Dimitrov, C. Y. Kuo, and M. S. Kokoska, "Ultrasound gel causes fine needle aspiration artifact? a clear choice," Acta Cytologica, vol. 56, no. 2, pp. 146-154, 2012.

[39] N. Arora, T. Scognamiglio, B. Zhu, and T. J. Fahey III, "Do benign thyroid nodules have malignant potential? an evidence-based review," World Journal of Surgery, vol. 32, no. 7, pp. 1237-1246, 2008.

[40] M. J. Jeon, D. E. Song, C. K. Jung et al., "Impact of reclassification on thyroid nodules with architectural atypia: from non-invasive encapsulated follicular variant papillary thyroid carcinomas to non-invasive follicular thyroid neoplasm with papillary-like nuclear features," PLoS One, vol. 11, no. 12, Article ID e0167756, 2016.

[41] S. Sung, E. Margolskee, D. Chen, and P. Tiscornia-Wasserman, "Incidence of noninvasive follicular thyroid neoplasm with papillary-like nuclear features and change in risk of malignancy for "The bethesda system for reporting thyroid cytology," Journal of the American Society of Cytopathology, vol. 8, no. 3, pp. 133-140, 2019. 